\title{
ON PRODUCT AND BUNDLE NEIGHBORHOODS
}

\author{
M. L. CURTIS ${ }^{1}$ AND R. K. LASHOF ${ }^{1}$
}

If a nice space $X$ is embedded in a euclidean space, it may fail to have product neighborhoods; i.e., neighborhoods which are products of $X$ with a ball. However, if the euclidean space is a hyperplane of a higher-dimensional euclidean space one can sometimes guarantee the existence of product neighborhoods in the big euclidean space. For example, it follows from a lemma due to Klee [1] that if $X$ is a $k$-ball in $R^{n} C R^{n+k}$, then $X$ has a neighborhood homeomorphic with $X \times B^{n}$ in $R^{n+k}$ (where $B^{n}$ denotes an $n$-ball). We obtain two results along these lines. Theorem 1 gives circumstances in which we get product neighborhoods and Theorem 2 yields ball-bundle neighborhoods. Theorem 2 has been used for smoothing combinatorial manifolds [3].

Definition. We say that a space $X$ has a local multiplication into a space $Z$ if there exists a neighborhood $N$ of the diagonal $\Delta$ of $X \times X$ and a map $\phi: N \rightarrow Z$ such that:

(i) $\phi(\Delta)=z_{0} \in Z$,

(ii) $\phi \mid N_{x}$ is one-to-one, and $\phi\left(N_{x}\right)$ contains a fixed neighborhood $W$ of $z_{0}$, for all $x$ in $X$. ( $N_{x}=$ pairs in $N$ with first coordinate $x$.)

EXAMPLE 1 . Any topological group $G$ has a local multiplication into itself. Take $N=G \times G$ and define $\phi(g, h)=g h^{-1}$.

EXAMPLE 2. If $X$ is a $k$-dimensional parallelizable manifold, then $X$ has a local multiplication into $R^{k}$. We take a Riemannian metric for $X$ and choose $N$ so that if $(x, y) \in N$, then there is a unique geodesic from $x$ to $y$. Let $\tau(x, y)$ denote the vector tangent to this geodesic at $x$ and having length equal to the length of the geodesic. Let $c$ be a cross section of the $k$-frame bundle over $X$. (c exists since $X$ is parallelizable.) We define $\phi(x, y)$ to be the point in $R^{k}$ with coordinates equal to the dot products of $\tau(x, y)$ with the vectors of $c(x)$. It is easy to verify that $\phi$ is a local multiplication.

A space $S$ is said to have the neighborhood extension property if for any closed subset $B$ of a separable metric space $Y$ and any map $f: B \rightarrow S$, there exists an extension of $f$ to some neighborhood of $B$.

THEOREM 1. Let $X$ be a compact space which has the neighborhood extension property and has a local multiplication $\phi$ into $Z$. Let $\alpha: X \rightarrow H$

Presented to the Society November 17, 1961, under the title $A$ note on product neighborhoods; received by the editors July 17, 1961 and, in revised form, October 27, 1961.

1 Partially supported by NSF Grants G-14089 and G-10369. 
be an embedding of $X$ into a locally compact separable metric group $H$. Then for any sufficiently small compact neighborhood $U$ of the identity $e$ of $H$, there exists a product neighborhood $X \times U$ of $\alpha(X)$ in $H \times Z$.

Proof. We will consider that $\alpha$ embeds $X$ into $H \times z_{0}$ and show that $\alpha$ can be extended to a homeomorphism $\psi$ of $X \times U$ into $H \times Z$. Here $z_{0}=\phi(\Delta)$.

Extend $\alpha^{-1}: \alpha(X) \rightarrow X$ to a map $\beta$ of a neighborhood $V$ of $\alpha(X)$ into $X$ ( $V$ is a neighborhood in $H$ ). Choose a compact neighborhood $U$ of $e$ in $H$ such that $U \cdot \alpha(X) \subset V$. Let $\tilde{x}=\alpha(x)$ and define

$$
\psi(x, h)=(h \tilde{x}, \phi\{\beta(h \tilde{x}), x\}) .
$$

One easily checks the following properties of $\psi$.

(1) $\psi(x, e)=\left(\tilde{x}, z_{0}\right)=\alpha(x)$,

(2) $\psi$ is one-to-one and on to a neighborhood.

(3) $\psi$ is continuous.

Since $X \times U$ is compact, $\psi$ is a homeomorphism and the theorem is proved.

CoRollary 1. If $G$ is a group with the neighborhood extension property and $G$ is embedded in $H$, then $G$ has small product neighborhoods $G \times U$ in $H \times G$.

Corollary 2. If $X$ is a parallelizable closed $k$-manifold in $R^{n}$, then $X$ has a product neighborhood $X \times B^{n}$ in $R^{n+k}$.

For example, this is the case if $X$ is a closed orientable 3-manifold or is a compact Lie group. Either Corollary 1 or 2 shows that a simple closed curve $S^{1}$ in $R^{n}$ has a product neighborhood $S^{1} \times B^{n}$ in $R^{n+1}$. For $k>1$, the fact that $S^{k} \subset R^{n}$ has a product neighborhood in $R^{n+k}$ follows from Klee's lemma and Stalling's unknotting theorem [4]. In general, we would like to show that a $k$-manifold $X$ in $R^{n}$ has an $n$ ball-bundle neighborhood in $R^{n+k}$. We cannot do this, but can get ball-bundle neighborhoods if we are willing to raise the dimension of the embedding space. The next theorem shows how this is done.

Let $X$ be a compact space. We assume the following about $X$ which will be automatically true if $X$ is a smooth manifold: (1) the diagonal $\Delta$ of $X \times X$ has a $k$-ball bundle neighborhood $U$ in $X \times X$. Precisely, we assume that there exists a $k$-plane bundle $\tau=\tau^{k}$ over $X(=\Delta)$ and a homeomorphism $g$ of $U$ onto the vectors of length less than or equal to one (for some metric in $\tau$ ) such that $g(x, y) \in \tau_{x}$, $(x, y) \in U, \tau_{x}$ the fibre over $x \in X .^{2}$ We identify $U$ with $\tau_{1}$. For $X$ a

\footnotetext{
${ }^{2} g(x, x)=0 \in \tau_{x}$
} 
smooth manifold the normal bundle of $\Delta$ (which is the same as the tangent bundle of $X$ ) gives such a neighborhood.

Since $X$ is compact there exists a vector bundle $\nu^{m}$ over $X$ such that $\tau^{k} \oplus \nu^{m}$ is a trivial bundle. (See (2.19) and (2.20) of [2].) Since $\tau^{k} \oplus \nu^{m}$ is trivial, we have a fibre-preserving homeomorphism $\phi: \tau^{k} \oplus \nu^{m} \rightarrow X \times R^{k+m}$, and for $x \in X$ we denote its restriction to the fibre $\tau_{x} \oplus \nu_{x}$ by $\phi_{x}$.

THEOREM 2. Suppose $X$ is compact, satisfies (1), and has the neighborhood extension property. If $\alpha: X \rightarrow R^{n}$ is any embedding of $X$ in $R^{n}$, then $\alpha(X)$ has a ball-bundle neighborhood in $R^{n} \times R^{k+m}$.

Proof. Let $0^{n}$ be the product $n$-plane bundle over $X$, and consider that $\alpha$ embeds the zero cross section of $0^{n} \oplus \nu^{m}$. We will prove that there exists $\epsilon>0$ and an embedding

$$
\psi: 0_{e}^{n} \oplus \nu^{m} \rightarrow R^{n} \times R^{k+m}
$$

which extends $\alpha$.

Let $\beta: N \rightarrow X$ be an extension of $\alpha^{-1}$ to a neighborhood $N$ of $\alpha(X)$. If $(x, h) \in X \times R^{n}$ and $\|h\|<\epsilon$, we can consider $(x, h) \in 0_{\epsilon}^{n}$. Let $\tilde{x}$ denote $\alpha(X)$ and choose $\epsilon$ small enough so that, for $\|h\|<\epsilon, \tilde{x}+h \in N$ and $(\beta(\tilde{x}+h), x) \in U$.

For $x, y \in X$ we define a map $f_{x y}: \nu_{x} \rightarrow \nu_{y}$ to be the composition

$$
\nu_{x} \stackrel{\text { incl }}{\longrightarrow} \tau_{x} \oplus \nu_{x} \stackrel{\phi_{x}}{\longrightarrow} R^{k+m} \stackrel{\phi_{y}^{-1}}{\longrightarrow} \tau_{y} \oplus \nu_{y} \stackrel{\text { proj. }}{\longrightarrow} \nu_{y} .
$$

We note that $f_{v x}$ is linear and if $(y, x)$ is in a sufficiently small neighborhood of $\Delta$ in $X \times X$, then $f_{y x}$ is an isomorphism. We assume $\epsilon$ is small enough so that $(\beta(\tilde{x}+h), x)$ is in such a neighborhood for $\|h\|<\epsilon$.

For notational convenience let $\sigma=\tilde{x}+h$ and $\rho=\beta(\tilde{x}+h)$. For $v \in \nu_{x}$ we define

$$
\psi((x, h)+v)=\left(\sigma, \phi\left\{(\rho, x)+f_{\rho x}(v)\right\}\right) .
$$

Then $\psi$ maps $0_{a}^{n} \oplus \nu^{m}$ into $R^{n} \times R^{k+m}$, and $\psi$ is clearly continuous. It remains to check that $\psi$ is one-to-one.

Suppose $\psi\left(\left(x_{1}, h_{1}\right)+v_{1}\right)=\psi\left(\left(x_{2}, h_{2}\right)+v_{2}\right)$. Then $\sigma_{1}=\sigma_{2}$ so $\rho_{1}=\rho_{2}$ and hence both $\left(\rho_{1}, x_{1}\right)$ and $\left(\rho_{2}, x_{2}\right)$ are in the fibre $\tau_{\rho_{1}}$. Similarly, both $f_{\rho_{1} x_{1}}\left(v_{1}\right)$ and $f_{\rho_{2} x_{2}}\left(v_{2}\right)$ are in the fiber $\nu_{\rho_{1}}$. Now $\phi$ is an isomorphism and these fibers are disjoint, so we must have $\left(\rho_{1}, x_{1}\right)=\left(\rho_{2}, x_{2}\right)$ so that $x_{1}=x_{2}$ and $h_{1}=h_{2}$. Also $f_{\rho_{1} x_{1}}$ is one-to-one so that $v_{1}=v_{2}$ and the theorem is proved.

REMARK 1. Theorem 2 yields Corollary 2 to Theorem 1 as a special 
case, because if $X$ is parallelizable then $\tau$ is trivial and $\nu$ is not needed. Hence $\psi$ embeds $X \times B^{n}$ into $R^{n} \times R^{k}$. More generally

Corollary 1. If the diagonal $\Delta$ of $X \times X$ has a $k$-ball bundle neighborhood $\tau_{1}$ such that $\tau$ is stably trivial (i.e., $\tau$ plus a trivial bundle is trivial), then $\psi$ embeds $X \times B^{n+1}$ in $R^{n} \times R^{k+1}$.

REMARK 2. The proof of Theorem 2 applies to an embedding $\alpha$ of $X$ into any smooth manifold $V^{n}$, if we replace $o^{n}$ by the tangent bundle $\tau^{\prime}$ of $M$ restricted to $X$. Then $\psi$ becomes an embedding of $\left(\tau^{\prime} \mid X\right)_{e} \oplus \nu^{m} \rightarrow V^{n} \times R^{k+m}$ which extends $\alpha$.

\section{REFERENCES}

1. V. L. Klee, Some topological properties of convex sets, Trans. Amer. Math. Soc. 78 (1955), 30-45.

2. John Milnor, Differential topology (Mimeographed notes) Princeton, N. J., 1958.

3. - Micro bundles and differentiable structures (Mimeographed notes), Princeton, N. J., 1961.

4. John Stallings, Piecewise-linear structures of higher-dimensional manifolds, to appear.

Florida State University and

University of Chicago 